\title{
Étude du lien entre l'échec à l'épreuve unique ministérielle en histoire et les difficultés dans le domaine du français
}

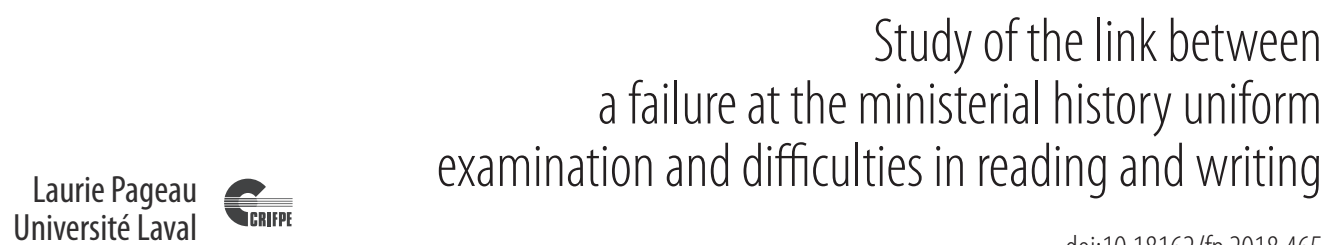

L'épreuve unique ministérielle en histoire (ÉUM) reposant en grande partie sur la mobilisation d'un savoir-lire et d'un savoir-écrire pour démontrer ses compétences en histoire, nous nous sommes intéressée à la relation entre l'échec à l'ÉUM et les difficultés éprouvées par l'apprenant dans le domaine du français (DF). Les dires de 102 participants inscrits à des cours de rattrapage d'été en histoire furent analysés : la majorité déclare éprouver des difficultés avec

la compétence « compréhension de textes » du DF. Lors de la démonstration de ses compétences en histoire, une mauvaise maîtrise des savoir-lire et savoir-écrire semble être une difficulté importante.

\section{Mots-clés}

Enseignement de l'histoire, savoir-lire, savoir-écrire, didactique de l'histoire, épreuve unique ministérielle, inférence.

Abstract

In Québec, the ministerial uniform examination of history (MUE) is based largely on the mobilization of reading and writing skills to demonstrate students' competency in history. This study focuses on the possible relationship between the failure of the MUE and the difficulties experienced by the learner in understanding and writing texts in French class. 102 students, attending summer remedial courses in history, answered our survey. Most of them declared having difficulty understanding written texts. When it's time to demonstrate history skills, poor literacy seems to be a problem.

\section{Keywords}

History education, literacy, writing skills, ministerial uniform examination.

\section{Introduction et problématique}

L'histoire est une science qui interprète le passé à la lumière des traces laissées par l'activité humaine à l'aide de la démarche historique qui permet la collecte et le traitement de l'information. Bien souvent, ces traces sont des documents textuels dont l'interprétation transite nécessairement par l'utilisation d'un savoir-lire. L'interprétation du contenu de ces documents se communique elle aussià l'aide d'un savoirfaire particulier : le savoir-écrire. Considérant l'importance relative de ces savoir-faire dans la démarche de l'historien professionnel, il est logique de les retrouver dans la classe d'histoire du secondaire et, au Québec, dans l'exécution de l'épreuve unique ministérielle d'histoire. Dans le présent article, nous tenterons d'esquisser un lien entre le manque de maitrise de ces savoir-faire chez certains élèves et le constat d'échec à l'épreuve unique d'histoire qu'ils ont obtenu.

En 2015, le taux de réussite à l'épreuve unique ministérielle d'histoire était de 82,3\% (Ministère de l'Éducation et de l'Enseignement supérieur, 2017a). Il est donc possible de déduire que le taux d'échec à l'épreuve unique ministérielle d'histoire était de 17,7\%. Il s'agit d'un pourcentage cohérent avec celui des années antérieures (Ministère de l'Éducation et de l'Enseignement supérieur, 2013, 2014; Ministère de l'Éducation, de l'Enseignement supérieur et de la Recherche, 2015a, 2015b). En appliquant ce pourcentage à l'ensemble de la population de quatrième secondaire inscrite en 2015 (Ministère de l'Éducation, de l'Enseignement supérieur et de la Recherche, 2015c), on peut estimer qu'environ 10271 élèves sont en position d'échec à la suite de la passation de l'épreuve unique ministérielle en histoire. Pour comprendre l'ampleur du problème, il faut considérer deux éléments : premièrement, l'épreuve unique, qui compte pour $50 \%$ de la note finale de quatrième secondaire en histoire et, deuxièmement, 
la réussite du cours d'histoire que sanctionne cette épreuve et qui est obligatoire pour l'obtention du diplôme d'études secondaires. Bon an, mal an, ce sont environ 10000 élèves par année qui, à la suite de l'obtention d'une mention d'échec en histoire, se trouvent dans une situation scolaire ardue, voyant leurs chances d'être admis dans un programme d'études collégiales ou un programme de formation professionnelle être compromises, sans parler des chances de décrocher un emploi. L'élève en échec en histoire se trouve donc dans une situation délicate menant, habituellement, à la reprise de l'épreuve unique.

Une des options possibles pour l'apprenant dans cette situation d'échec est de suivre un cours de rattrapage durant la saison estivale. En effet, le ministère offre aux élèves de s'inscrire à une reprise de l'épreuve unique en août. Pour s'y préparer, les commissions scolaires proposent un service ponctuel et privé de rattrapage et de préparation pour l'épreuve unique. Nous tenons à préciser qu'il s'agit bien d'un cours de préparation à l'épreuve unique plutôt que d'une reprise du cours d'histoire dans sa totalité; ainsi, certaines compétences, présentes dans le programme d'histoire, sont complètement évacuées du programme des cours d'été puisqu'elles ne sont pas évaluées à cette épreuve ${ }^{1}$.

L'épreuve ministérielle transite actuellement par une évaluation écrite. Considérant les impératifs inhérents à l'administration d'une épreuve certificative des compétences à l'ensemble de la province du Québec, soit environ 58027 élèves en 2015, il serait difficile de faire autrement. Pour les élèves ayant des difficultés dans le domaine du français, il est possible que cette façon de procéder nuise à la réussite de l'épreuve. En effet, puisque l'épreuve unique d'histoire évalue la compétence « interroger les réalités sociales dans une perspective historique $»^{2}$, lélève doit, à l'aide de documents historiques (la plupart étant des textes), être capable de démontrer sa maîtrise de cette compétence. Cela implique d'être apte à comprendre la question formulée à l'écrit, puis d'aller chercher dans les documents d'accompagnement (majoritairement textuels) les éléments de réponse requis pour pouvoir formuler une réponse qui transitera, elle aussi, par l'écrit.

En 2003, une enquête internationale a révélé que près de la moitié de la population canadienne de 16 à 35 ans n'a pas les compétences requises pour comprendre des textes schématiques ou suivis, pour utiliser efficacement de l'information ou avoir une participation active à la collectivité (Blaser, 2007; Grenier et al., 2003). Considérant que l'épreuve unique d'histoire transite en grande partie par l'application de la méthode historique à des textes écrits ${ }^{3}$, on peut apprécier l'effort considérable demandé à certains des élèves qui se classeraient parmi cette population ayant de la difficulté à utiliser efficacement l'information écrite.

Considérant cela, nous en venons à nous demander si l'évaluation de la compétence «interroger les réalités sociales dans une perspective historique » du domaine de l'histoire ne reposerait pas, en partie, sur la maîtrise d'un savoir-écrire et d'un savoir-lire qui sont particulièrement développés par certaines compétences issues du domaine du français, soit les compétences de rédaction et de compréhension de textes. C'est ainsi qu'au cours d'une recherche voulant documenter la perception des causes de leur échec à l'épreuve unique ministérielle des apprenants inscrits à des cours d'été (Pageau, 2016), nous avons documenté le lien existant entre l'échec à l'épreuve unique ministérielle et les difficultés éprouvées dans le domaine du français. Au vu de ces divers éléments de nature à compliquer et entraver la démonstration des compétences du domaine de l'histoire transitant par le savoir-lire et le savoirécrire mobilisés lors de l'exécution de l'épreuve unique ministérielle, nous nous posons la question 
de recherche suivante : existerait-il un possible lien entre les difficultés lourdes ressenties dans les compétences de compréhension de textes et de rédaction de textes issues du domaine du français (qui mobilisent particulièrement le savoir-lire et le savoir-écrire) et l'échec à l'épreuve unique histoire?

\section{Note, avant de poursuivre}

Certaines recherches désignent le savoir-lire par le terme de « compétence lectoriale » (voir, notamment, Chartrand, 2009). Pour éviter toute confusion avec les compétences disciplinaires auxquelles nous nous référerons, nous avons préféré employer les concepts de "savoir-lire » et "savoir-écrire » dans le cadre de cet article. Cela permettait d'insister sur la mobilisation de ces savoirs pour être capable de démontrer sa maîtrise des compétences en histoire. Nous emploierons volontairement le terme de «compétence » pour désigner ce qui est développé, respectivement, dans les cours d'histoire et de français et nous utiliserons "savoir-faire » (particulièrement "savoir-lire » et "savoir-écrire ») pour insister sur l'aspect transversal de ces acquis.

\section{Cadre conceptuel}

L'acte de lire est fondamental pour les historiens. L'analyse, la compréhension et la mise en contexte des archives sont au cœur de la réflexion en histoire. Une fois ces processus achevés vient le moment du partage des réflexions et des découvertes transitant le plus souvent par l'écrit. Le travail de l'historien demande donc l'utilisation d'un savoir-lire et d'un savoir-écrire adaptés à son domaine de recherche (Wineburg, 1991; Wineburg et Martin, 2004; Wineburg, Martin, et Monte-Sano, 2011). S'il est possible, dans la classe, d'utiliser la pensée historique sans transiter par le savoir-lire et le savoir-écrire (voir Demers, Lefrançois et Éthier, 2014 sur ce sujet), dans un contexte d'évaluation ministérielle, ces deux savoir-faire sont très sollicités (Déry, 2017). Pour mieux comprendre l'importance de ces savoirfaire, nous nous attarderons, dans la présente section, à décrire l'épreuve unique puis nous élaborerons sur des causes possibles de difficultés pouvant survenir à l'épreuve unique, soit les problèmes associés à l'abstraction en écriture appliquée au domaine de l'histoire, la nature particulière des concepts en histoire, les spécificités textuelles de cette discipline, la présence d'inférences dans l'épreuve unique et la médiation par le langage écrit omniprésent dans l'épreuve unique. Tous ces éléments, qui peuvent sembler disparates, ont un rôle à jouer pour expliquer le lien entre le savoir-lire, le savoir-écrire et l'échec dans le domaine de l'histoire.

\section{La structure de l'épreuve unique}

La structure de l'épreuve unique se divise deux sections. Une première est composée de 21 questions à réponses courtes regroupées selon les quatre thématiques du programme d'histoire de deuxième cycle du secondaire (qui sont, rappelons-le : Pouvoir et pouvoirs; économie et développement; population et peuplement; culture et mouvements de pensée). Pour chaque thématique, une dizaine de documents sont fournis à l'élève (soit environ 40 documents pour l'ensemble de cette section). Cette section est notée sur 38 points (sur un total de 50 , soit $76 \%$ de l'épreuve) et cherche à évaluer des opérations intellectuelles que l'apprenant effectue sur les sources fournies. La deuxième portion de l'épreuve est 
une question à développement, cherchant à évaluer la rigueur du raisonnement de l'apprenant, ayant une valeur de 12 points sur un total de 50 (soit $24 \%$ de l'épreuve). La structure attendue de ce texte est librement inspirée de la dissertation historique et elle requiert de l'apprenant qu'il réponde à une question donnée à l'aide de deux ou trois arguments appuyés de faits historiques qui doivent être obligatoirement issus de la dizaine de documents fournis dans le dossier documentaire ${ }^{4}$. Cette façon de procéder permettrait d'évaluer la pensée et la méthode historique. Avant de poursuivre plus loin dans notre argumentaire, nous définirons ici sommairement la pensée historique : l'historien, lors de la consultation des sources premières et secondaires à sa disposition, met en place un processus d'analyse critique, de tri et de classement de l'information qui lui permettent de construire son interprétation des phénomènes qu'il étudie. Les opérations intellectuelles utilisées lors de cette analyse sont ce que nous désignons par le terme de pensée historique. Les cours d'histoire au primaire et au secondaire visent à familiariser lélève avec cette pensée historique. Warren résume très bien ce qui est attendu de l'apprenant lors de l'épreuve unique cherchant à évaluer la maîtrise de cette pensée historique : "[1]'élève devait se servir de ces documents pour réaliser des opérations intellectuelles dont ils devenaient en quelque sorte le prétexte. L'important, c'était d'abord de pouvoir déterminer des facteurs explicatifs et des conséquences, d'établir des liens de causalité ou de mettre en relation des faits. » (2013, p. 45). Si cette structure permet, théoriquement, de mettre en place toutes les conditions pour évaluer l'utilisation de la pensée historique, elle pourrait aussi être contournée par l'apprenant ayant des forces dans d'autres stratégies et habiletés transversales que celles du domaine d'histoire (nous reviendrons sous peu sur cette idée).

\section{Quelques éléments à considérer pour comprendre le lien entre l'échec en histoire et les compétences du domaine du français}

Pour mieux comprendre le lien qui s'esquisse entre les difficultés dans le domaine de l'histoire et celui $\mathrm{du}$ français, il faut prendre en considération, entre autres, le genre textuel particulier du domaine de l'histoire, un genre caractérisé par des schémas d'écriture complexes à déchiffrer pour un lecteur adolescent (Chartrand, 2009; Ciardiello, 2002; Nokes, 2013; Wineburg, 2001; Wineburg et al., 2011) et le lexique particulier à la discipline historique (Chartrand, 2009; Laparra, 1991; Moniot, 1993). Ces éléments entraînent le besoin d'apprendre à l'élève à lire en histoire, impliquant ce que Martel (2014) désigne comme un savoir-lire transversal qui serait mobilisable dans plusieurs disciplines. Appliqué dans le domaine de l'histoire, ce savoir-lire implique un constant aller-retour entre les questionnements et les sources (Demers et al., 2014; Martel, 2014; Nokes, 2013; Wineburg et Martin, 2004; Wineburg et al., 2011). L'écriture implique une construction de sens et de structuration de l'expérience et, en plus de mobiliser un savoir-lire, l'évaluation de l'emploi par l'élève de la méthode historique implique la mobilisation d'un savoir-communiquer permettant de démontrer le résultat du cheminement intellectuel de l'apprenant (Monte-Sano, 2011; Stan, 2017, p. 35; Wineburg et Martin, 2004). Dans le cadre de l'épreuve unique, le «savoir-communiquer » mobilisé est le savoir-écrire. Encore une fois, il s'agirait d'un savoir-écrire transversal, qui peut être mobilisé dans plusieurs domaines d'études, chacun avec des particularités qui leur sont propres ${ }^{5}$. Cette idée est cohérente avec Wineburg (Wineburg et Martin, 2004; Wineburg et al., 2011) et Nokes (2013) qui considèrent qu'au cœur de l'enseignement de l'histoire devraient se trouver la lecture et l'écriture historique. 
En plus de ces savoir-faire transversaux, certains autres éléments doivent être pris en considération pour comprendre le lien qui peut s'établir entre les difficultés dans le domaine de l'histoire et celles du domaine du français. Nous ferons ici la liste de six éléments permettant de comprendre ce lien.

\section{L'abstraction liée à l'écriture}

Le geste d'écrire implique de formuler en phrases complètes un langage intérieur composé, entre autres, d'émotions, d'intuitions ainsi que de sentiments pour être intelligible par un interlocuteur extérieur (Blaser, 2007; Schneuwly, 2008). Cela requiert que l'apprenant puise dans sa capacité d'abstraction pour formuler sa pensée, mais aussi, pour prévoir la réaction de son interlocuteur. En effet, en histoire, la tâche requise lors de la rédaction de la question longue est complexe : pour l'élève, avoir à convaincre par écrit son interlocuteur/correcteur qu'il a bien compris la question, la période historique, les textes du dossier documentaire, les arguments sous-entendus dans ces textes, le tout en démontrant la rigueur de son raisonnement en répondant à une question historique requiert un très haut niveau de maîtrise de l'abstraction.

\section{La nature des concepts en histoire}

La structure actuelle de l'épreuve unique ne permet pas de se contenter de mémoriser la définition de certains concepts à l'étude pour les restituer de manière boulimique à l'épreuve unique. L'apprenant doit pouvoir reconnaître ces concepts lorsqu'ils sont employés dans des documents historiques. Ces concepts «ne peuvent se construire par la simple répétition ou mémorisation. Leur construction par les élèves exige un intense travail cognitif et langagier indissociable de la production d'écrits " (Blaser, 2007, p. 32). Déry $(2016,2017)$ dans son analyse de la posture épistémologie de l'épreuve unique d'histoire, conclut que l'épreuve unique sollicite peu la pensée historienne et repose plus sur une idée de reproduction du savoir transmis par l'utilisation d'un savoir mémorisé ou par l'utilisation d'une autorité (le document) pour réactiver des connaissances antérieures (pour les élèves maîtrisant suffisamment le savoir-lire pour pouvoir le faire). Alors que les concepts mobilisés en histoire devraient être le fruit d'un travail cognitif tel que mentionné par Blaser (2007), Déry $(2016,2017)$ indique que ce qui se vit à épreuve unique est tout autre. Dans une situation idéale, la maîtrise de ces concepts devrait être telle que l'apprenant puisse les reconnaître, puis classer l'information fournie pour ensuite planifier la production requise lors de l'évaluation (formative ou évaluative) pour démontrer l'acquisition du concept et, ultimement, de la compétence évaluée. C'est ce que Monte-Sano (2011) ainsi que Chartrand et Blaser (2007) classent comme des compétences de lecture et d'écriture de haut niveau.

\section{Les spécificités textuelles de la discipline}

Selon Blaser et Chartrand (2009) de même que Chartrand et Blaser (2007), tous les enseignants qu'elles ont interrogés reconnaissaient qu'il existe des écrits spécifiques à la discipline historique. Il a aussi été montré que l'enseignement de ces spécificités textuelles, entre autres la dissertation historique utilisée dans l'épreuve unique, semblait mettre mal à l'aise les enseignants d'histoire (Blaser et Chartrand, 2009; Blaser, Lord, Giraldeau et Trudeau, 2014; Chartrand et Blaser, 2007; Hall, 2005). Considérant que les 
élèves sont évalués sur la rigueur de leur raisonnement dans le cadre d'une production écrite inspirée du genre de la dissertation historique, il est inquiétant de constater ce malaise chez les enseignants.

\section{L'inférence}

La structure des questions employées dans l'épreuve unique repose beaucoup sur les capacités à faire des liens entre les questions d'examen et les documents historiques fournis, ainsi qu'à mobiliser des concepts préalablement développés en classe. Cette façon de procéder sollicite la capacité à faire des inférences de la part des élèves (définie ici comme la capacité à faire des liens entre des suggestions et des éléments de réponses possibles [Kendeou, van den Broek, Helder et Karlsson, 2014; Makdissi et Boisclair, 2008; Nokes, 2013] ou, autrement formulée, la capacité de lire entre les lignes). Or, les étudiants ayant des difficultés à décoder les textes ont tendance à compenser leurs faiblesses à l'aide de leur bagage de connaissances générales sur le sujet (Nokes, 2013, p. 92). Dans le domaine de l'histoire, considérant que les apprenants qui ont des difficultés de décodage sont aussi en train de se construire un bagage de connaissances générales, il se crée ici un cercle sans issue : sans connaissances générales pour compenser les difficultés de décodage, l'apprenant arrive difficilement à faire les inférences permettant l'acquisition de nouvelles connaissances historiques, ce qui rend l'apprenant dépendant de la mémorisation du récit qui deviendra son équivalent de bagage de connaissances générales historiques (voir Figure 1)

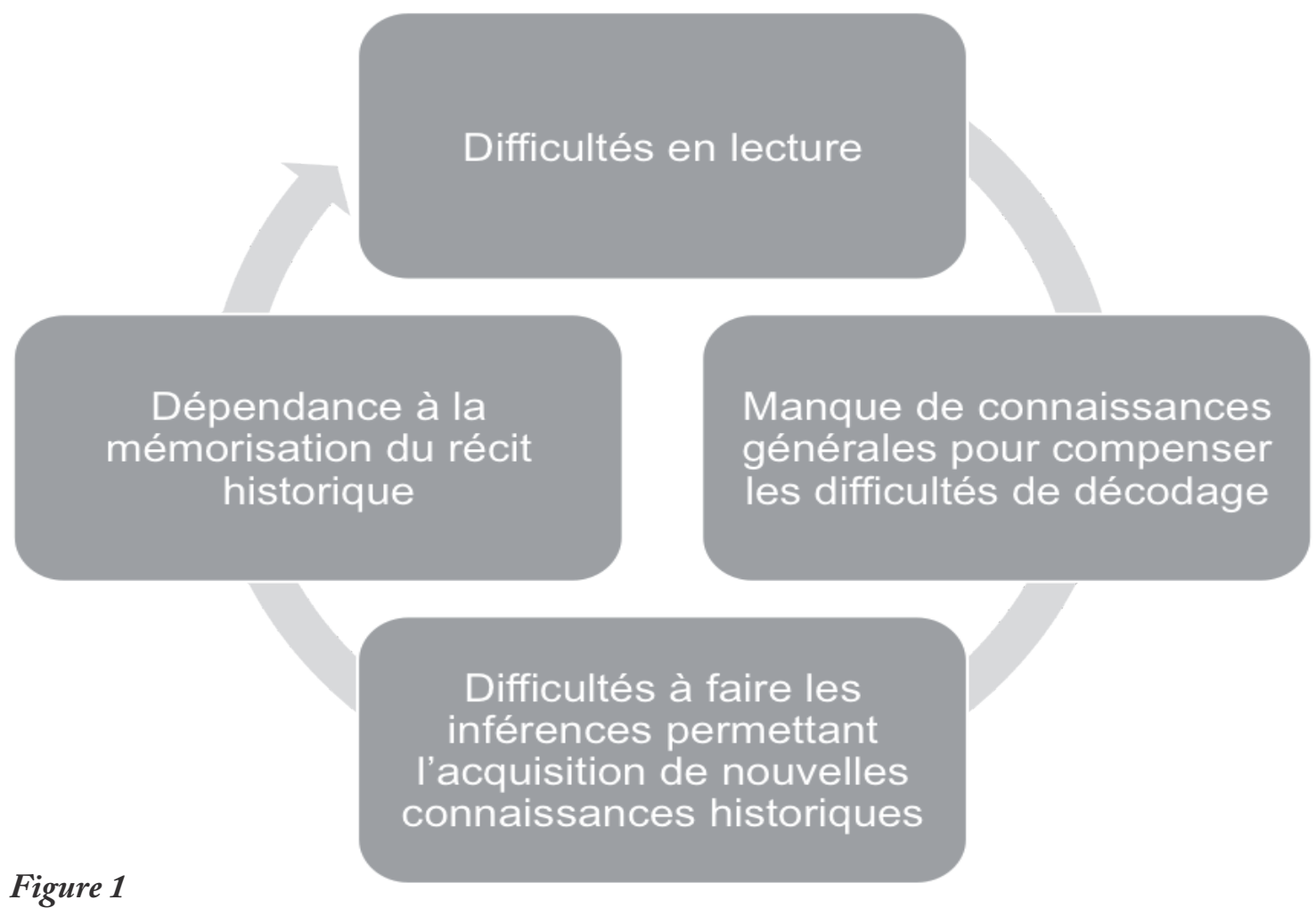

Schéma illustrant le lien entre les difficultés en lecture et la dépendance à la mémorisation en histoire. 
Lorsque les difficultés à faire des inférences se maintiennent jusqu'à l'épreuve unique, où il est impossible de connaître par cœur l'ensemble du récit et les détails qui seront à l'examen, l'apprenant en difficulté a peu de chance de réussite.

\section{La médiation par le langage écrit}

La médiation est un concept élaboré par Vygotski pour décrire la capacité de transformation d'un milieu (ou du savoir) par un outil. C'est ainsi qu'en apprenant un langage, on se dote d'un outil pouvant transformer notre pensée, mais aussi l'environnement autour de soi (Smidt, 2009). Dans l'exécution de l'épreuve unique, plusieurs médiations de l'outil qu'est le langage écrit entrent en jeu. C'est ainsi qu'un apprenant maîtrisant mal le langage écrit doit surmonter trois moments « critiques » à chaque question de l'épreuve unique (voir Figure 2). Un premier survient lors de la lecture de la question de l'examen dont lélève doit comprendre les demandes et subtilités (comme les questions à plusieurs volets et les questions demandant de justifier sa réponse). Un deuxième moment « critique » survient lors de l'observation et l'analyse des documents du dossier documentaire. Finalement, un troisième, lors de la rédaction (courte ou longue) de sa réponse écrite, où il doit transmettre à l'aide du langage écrit la réponse qu'il a élaborée au problème posé, en prenant en compte le contexte d'écriture, les informations à transmettre et le positionnement qu'il doit prendre.

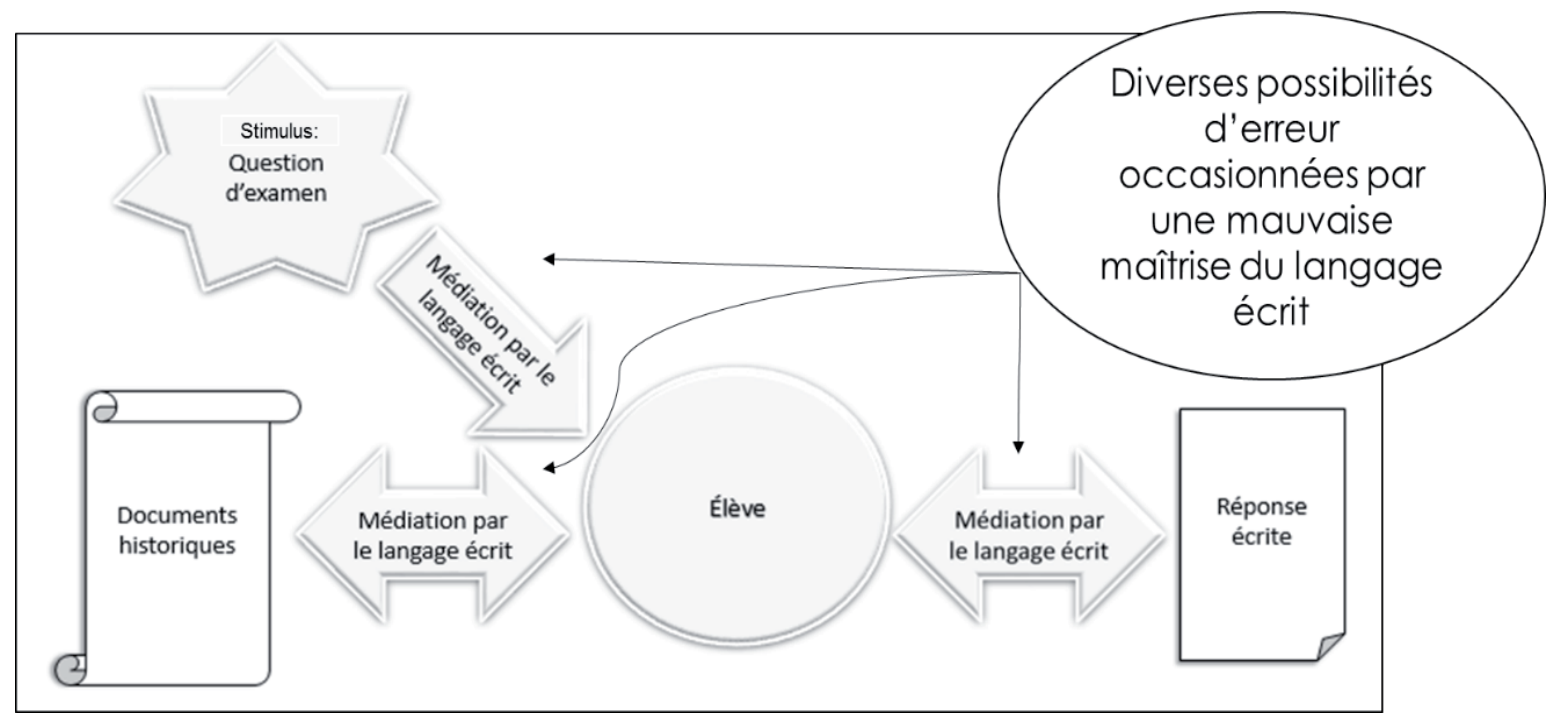

Figure 2

Schéma illustrant les possibilités d'erreur occasionnées par une mauvaise maîtrise du langage écrit.

À chacun de ces moments, une mauvaise compréhension ou utilisation de l'outil sémiotique peut mener à l'introduction d'une erreur dans la démarche et la réflexion de l'apprenant.

Il n'est pas attendu ici à ce que les participants à notre recherche exploratoire puissent identifier un de ces éléments comme étant la cause de son échec. Nous nous sommes attardée sur ceux-ci dans le but de permettre au lecteur d'avoir en tête ces éléments pour comprendre les difficultés qui seront 
exprimées par nos participants dans leurs propos. Bien que nous soyons en accord avec une évaluation en histoire qui ne soit pas qu'un simple exercice de mémorisation, nous voulons démontrer à quel point la tâche demandée à l'apprenant est complexe. L'apprenant doit mobiliser des savoir-faire en lecture et en écriture de haut niveau pour parvenir à maîtriser et démontrer ses compétences dans le domaine historique.

\section{Méthodologie}

Notre recherche documentait la perception qu'ont les élèves de leur échec en histoire (à l'épreuve unique et dans leur cours de quatrième secondaire, considérant comment les deux sont intimement liés), nous nous sommes donc inspirée de la recherche phénoménologique (Fortin, Côté et Filion, 2006; SavoieZajc, 2007) et de l'interactionnisme symbolique (Poisson, 1992). Pour documenter cette perception de leur échec, les participants approchés furent ceux inscrits dans un programme de rattrapage de l'examen d'histoire durant l'été (ce cours de rattrapage se conclut avec la reprise de l'épreuve unique ministérielle en août). Cela nous a permis d'interroger exclusivement des élèves ayant obtenu un constat d'échec à l'épreuve unique. Lors de cette étude exploratoire, un volet ${ }^{6}$ fut consacré à établir s'il existait un lien possible entre l'échec à l'épreuve unique et les difficultés dans le domaine du français, particulièrement dans les compétences de compréhension de textes et de rédaction de textes.

La collecte de données s'est effectuée dans quatre commissions scolaires de la grande région de Québec, pendant les étés 2014 et 2015. Trois des milieux approchés offraient des cours en présentiel et un quatrième offrait des cours à distance via Internet, cours ouverts à l'ensemble de la province du Québec. Le questionnaire d'enquête fut donc produit sur deux formats, soit sur papier (pour les participants fréquentant un lieu scolaire physiquement atteignable) et en version électronique (à l'aide de l'outil LimeSurvey) pour les participants virtuels. L'utilisation de ces deux outils a permis de collecter les propos de 160 répondants, soit $62 \%$ de la population inscrite à un cours de rattrapage en histoire dans ces commissions scolaires.

À la suite de nos observations préalables sur le terrain et de nos échanges avec les professionnels du milieu, l'outil d'enquête a été conçu en considérant qu'il allait être utilisé auprès d'une population de participants ayant fort probablement des besoins particuliers, allant des troubles dys ${ }^{7}$ ou évoluant avec des troubles d'attention. Considérant cela, notre outil contenait une vingtaine de questions courtes et simplement formulées, alternant entre les questions ouvertes et fermées (permettant à la fois de s'assurer de la cohérence des propos entre les déclarations dans les questions ouvertes et fermées et permettant aussi l'association aux divers éléments pouvant expliquer l'échec en histoire décrits dans le cadre conceptuel). Lors de l'administration en présentiel, toutes les questions furent lues à voix haute. Dans le cadre du présent article, nous nous intéresserons particulièrement aux éléments de réponses issus de deux questions fermées et d'une ouverte traitant spécifiquement du lien entre les difficultés dans le domaine du français et celles dans le domaine de l'histoire. 


\section{Les différences entre le milieu virtuel et présentiel}

Deux différences ont pu être notées entre les réponses provenant de l'outil virtuel et celles émanant de la version "papier ». Premièrement, les répondants du milieu virtuel ont été plus volubiles, utilisant beaucoup plus de lignes dans les réponses à questions ouvertes, alors que les participants du milieu en présentiel ont, eux, résumé leurs pensées en quelques phrases. Il est possible que la différence entre les milieux où fut effectuée la collecte (soit une salle de classe remplie de pairs par rapport à un lieu d'études virtuel qui était probablement à la maison) puisse expliquer cette disparité (Mialaret, 2004). Deuxièmement, le taux de participation a été beaucoup plus faible pour le milieu virtuel, soit $30 \%$, alors qu'en présentiel il a atteint $78 \%$. Nous avons attribué cette différence au fait que le participant virtuel devait quitter son lieu d'études habituel (le site web de la commission scolaire) pour être redirigé vers la page dédiée au questionnaire en ligne. Malgré ces quelques différences, la similitude des éléments soulevés et des expériences vécues par les répondants nous ont permis d'analyser ces données comme un seul même corpus.

Parmi les 160 répondants ayant participé à notre recherche, 102 ont participé au volet sur le lien entre français et histoire, celui-ci ayant été développé lors de la deuxième année de la recherche. Il s'agit d'un échantillon multicas répondant aux critères (Savoie-Zajc, 2007) suivants : avoir obtenu un constat d'échec à l'épreuve unique ministérielle, être inscrit au cours d'été d'une des quatre commissions scolaires de la région de Québec participantes et avoir obtenu l'autorisation parentale (ou offrir la sienne si le participant est adulte) pour la participation à l'étude. Cette cohorte de répondants est composée à $64 \%$ de jeunes femmes. Les participants ont entre 15 et 18 ans, ce qui en fait un groupe légèrement plus âgé qu'une population habituelle d'élèves de quatrième secondaire.

\section{Résultats}

\section{Quantitatifs}

Tous les élèves présents au cours d'été avaient obtenu un constat d'échec à l'épreuve certificative d'histoire. Considérant cela, nous avons déduit qu'ils avaient des difficultés de natures diverses dans le domaine de l'histoire. Nous avons interrogé la population présente en leur demandant d'évaluer s'ils avaient eu de lourdes difficultés à surmonter dans le domaine du français en compréhension de textes et en rédaction de textes. Pour nous assurer d'une bonne compréhension du concept de "difficulté lourde ", nous avons donné en exemple aux participants le fait d'avoir besoin d'un tuteur à domicile, d'avoir eu recours à des services de récupération à l'école durant une longue période de temps ou bien d'avoir obtenu un échec dans une ou les deux compétences du domaine du français ciblées dans les deux années scolaires précédentes. Les réponses obtenues nous permettent d'évaluer la perception qu'ont les répondants de leurs difficultés ${ }^{8}$ (voir Tableau 1). 


\section{Tableau 1}

Lien entre les difficultés en rédaction et en comprébension de textes.

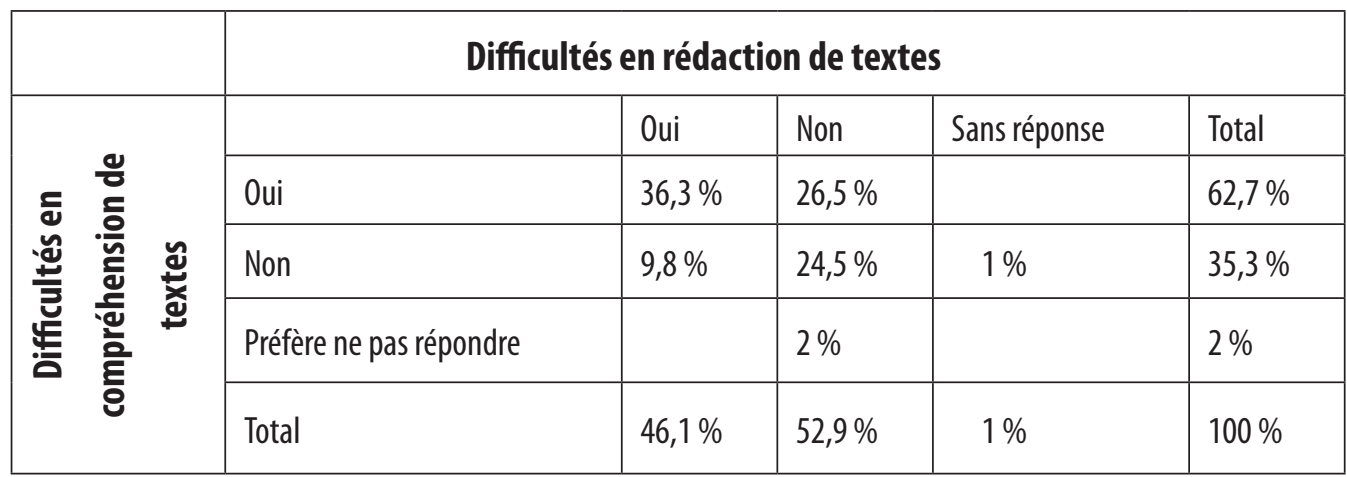

Parmi les 102 participants ayant répondu à ce volet du questionnaire, 62,7\% ont déclaré avoir des difficultés en compréhension de textes et $46,1 \%$ ont évalué avoir des difficultés en rédaction de textes. Un croisement des données permet d'évaluer que 36,3\% des répondants considèrent avoir des difficultés dans ces deux compétences (voir Figure 3). Il est plus fréquent qu'un élève déclare avoir de la difficulté en compréhension de textes sans avoir de difficulté en rédaction de textes que l'inverse.

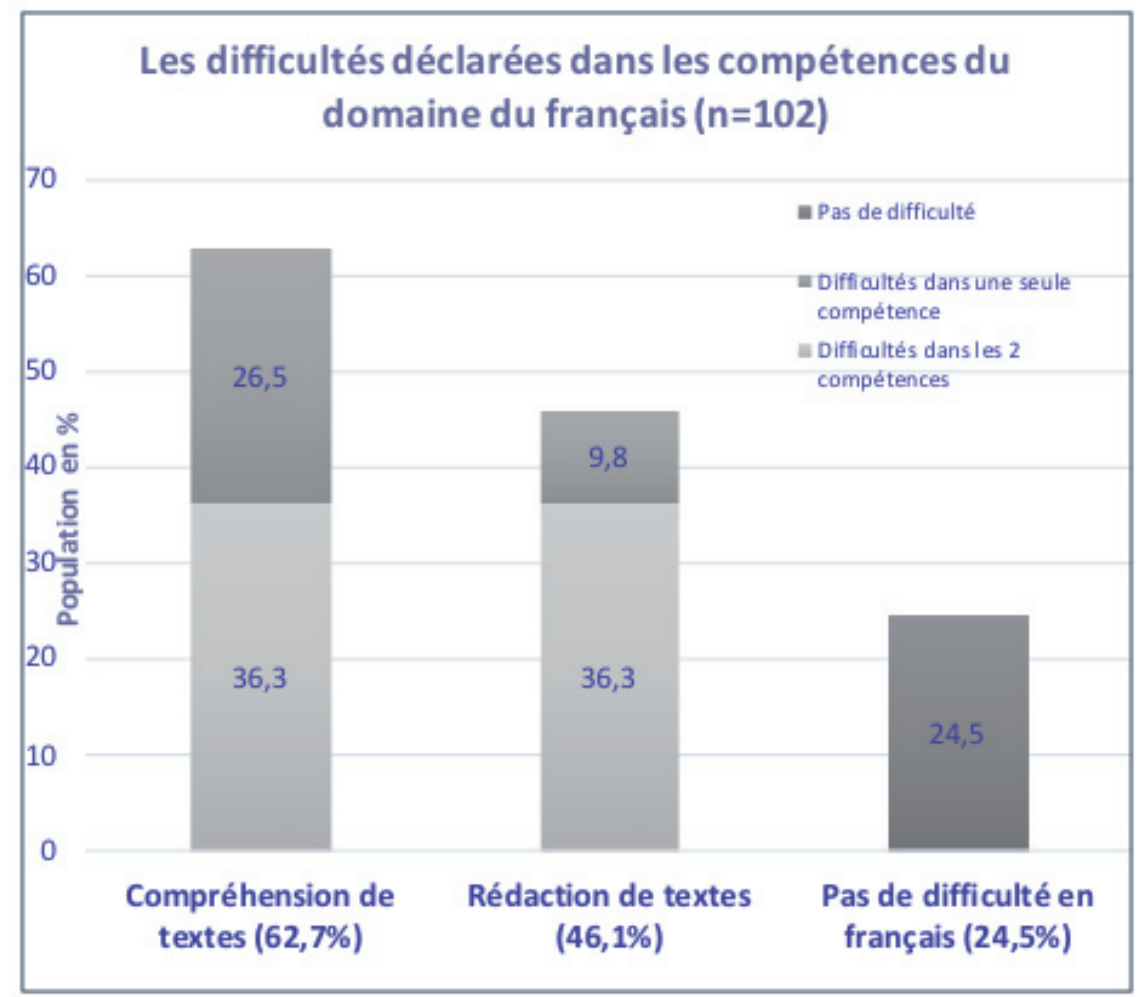

Figure 3

Les difficultés déclarées dans les compétences du domaine du français (n=102). 
Seulement 24,5\% des élèves ayant eu un constat d'échec à l'épreuve unique considèrent ne pas avoir de difficultés lourdes dans ces deux compétences de français.

\section{Qualitatifs}

Dans un deuxième temps, nous avons interrogé l'ensemble des 102 participants à ce volet de l'étude pour connaître leur opinion au sujet des compétences et connaissances développées dans le domaine du français pouvant être mobilisables en histoire à l'aide d'une question ouverte formulée ainsi : « D'après toi, en quoi tes acquis du cours de français peuvent-ils aider en histoire? » (voir Figure 4 et Tableau 2). Parmi nos participants, 97 ont répondu à cette question.

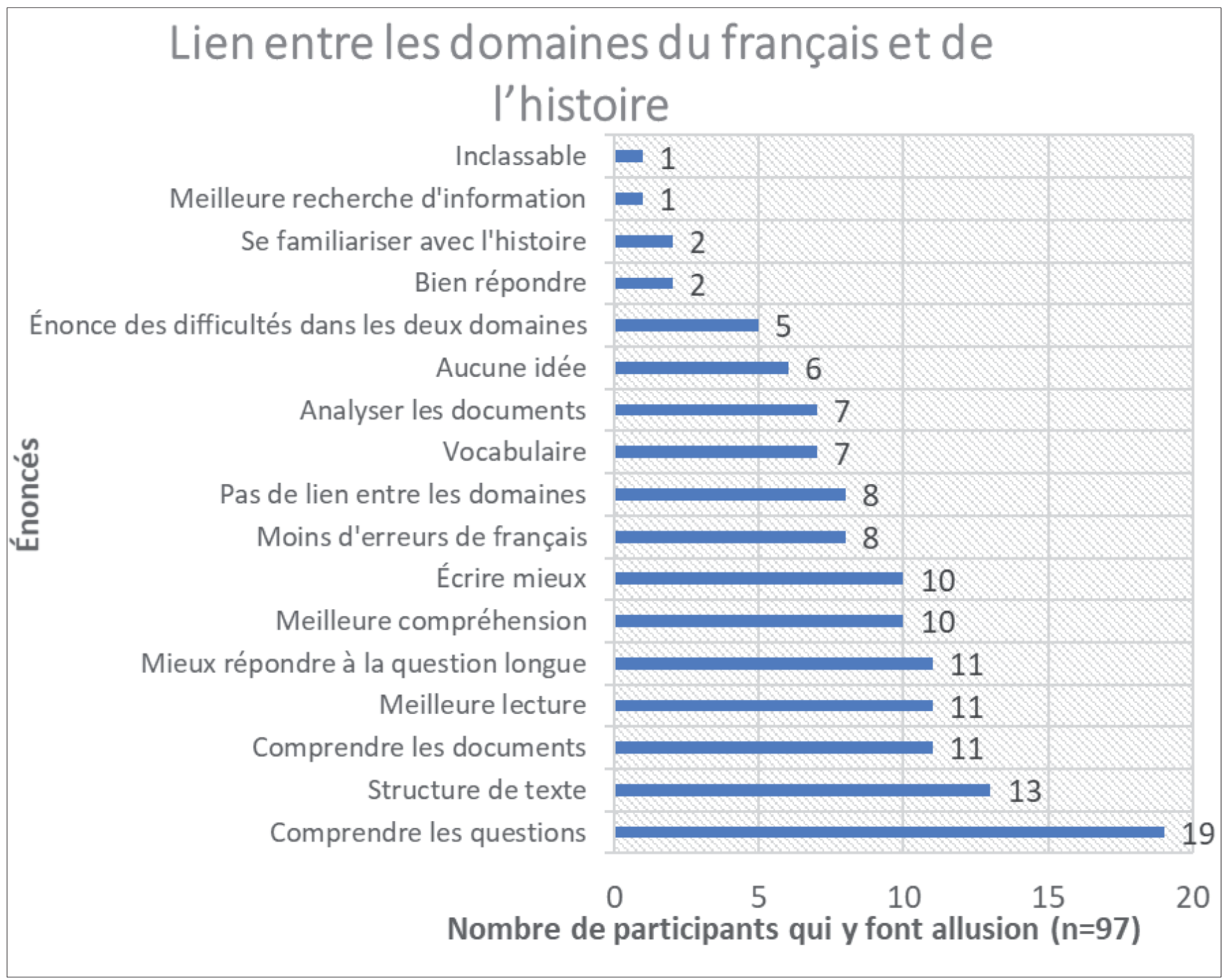

Figure 4

Liens mentionnés entre les domaines du français et de l'histoire. 
Ce graphique nous permet de visualiser les acquis du domaine du français que les répondants considèrent comme transférables en histoire. L'idée que les apprentissages en français peuvent les aider à comprendre les questions de l'épreuve unique est celle qui est la plus mentionnée. Puis, vient l'idée que ces acquis peuvent aider à structurer leur réponse (à développement ou non). Ils considèrent aussi que le domaine du français peut les aider à mieux comprendre les documents du dossier documentaire, que les stratégies de lecture peuvent leur être utiles dans le cours d'histoire et que les rédactions effectuées dans la classe de français peuvent les aider à mieux répondre à la question à développement en histoire.

\section{Tableau 2}

Quelques exemples de propos tenus par les répondants (sans révision) à la question: "D'après toi, en quoi tes acquis du cours de français peuvent-ils aider en histoire? ".

\begin{tabular}{ll} 
Sujet & Propos tenus \\
\hline Sujet \#3 & "Pour les questions de lecture, mieu [sic] comprendre ce qu'on lit. » \\
\hline Sujet \#11 & "de [sic] mieux lire et comprendre les questions et mieux s'exprimer en [sic] ecrit [sic] » \\
\hline Sujet \#96 & $\begin{array}{l}\text { "Lorsque nous devons développer sur certains sujet [sic]. Le Français [sic] aide à formuler des phrases qui ont } \\
\text { de l'allure et à faire moins d'erreurs! » }\end{array}$ \\
\hline Sujet \#102 & "a [sic] mieux m'exprimer de façon plus évoluer [sic] si on veut et a [sic] mieux comprendre les questions. »
\end{tabular}

Il est donc possible de conclure que 62,7 \% des participants ayant échoué à l'épreuve unique ministérielle et ayant participé à cette étude évaluent avoir des difficultés avec la compétence de compréhension de textes alors que 46,1 \% des répondants mentionnent des difficultés avec la compétence de rédaction de textes. Considérant que nos réponses émanent d'une population n'ayant pas un vocabulaire aussi précis sur les compétences du domaine du français que les chercheurs et les praticiens, nous avons regroupé les codes employés en deux grandes catégories, soit : les mentions d'éléments pouvant être associés au savoir-lire (incluant : analyse de document, comprendre les documents, meilleure lecture, meilleure compréhension et meilleure recherche d'information) ou au savoir-écrire (incluant : écrire mieux, mieux répondre à la question longue et structure de textes). Avec de tels regroupements, on s'aperçoit, premièrement, qu'il n'y a que 39 répondants sur une possibilité de 102 qui mentionnent des liens entre les acquis développés en classe de français et les gestes à poser pour comprendre un texte historique et, deuxièmement, qu'il n'y a que 30 cas qui font allusion à des éléments de savoir-écrire qui pourraient être réinvestis dans la classe d'histoire.

Certains répondants ne semblent pas percevoir le lien entre les compétences du domaine du français et celles de l'histoire : en effet, 8 mentionnent explicitement qu'il n'y a pas de lien, 7 écrivent que le français ne servait qu'à enrichir le vocabulaire, 8 considèrent que le français sert à faire moins d'erreurs (certains allant même jusqu'à mentionner que les erreurs de français étaient cumulées à l'épreuve unique d'histoire, ce qui éclaire leur faible compréhension de l'épreuve unique). 
Les élèves se déclarant en difficulté (en rédaction ou en compréhension) semblent plus sensibles au lien entre l'histoire et le français. En effet, 12 répondants (sur les 19 au total) ayant mentionné que les acquis du domaine du français aidaient à mieux comprendre les questions de l'épreuve d'histoire ont aussi déclaré avoir des difficultés lourdes en compréhension de textes. Les tableaux 3 et 4 nous permettent de visualiser que cette observation semble être valable pour plusieurs liens établis par les participants.

\section{Tableau 3}

Fréquence des liens entre les domaines de l'bistoire et du français mentionnés chez la sous-population des élèves en difficultés en comprébension de textes

\begin{tabular}{lcc}
\hline $\begin{array}{l}\text { Liens mentionnés entre les } \\
\text { domaines du français et de } \\
\text { I'histoire }\end{array}$ & $\begin{array}{l}\text { Fréquence du code chez } \\
\text { l'ensemble des répondants }\end{array}$ & $\begin{array}{l}\text { Fréquence du code chez les élèves déclarant } \\
\text { avoir des difficultés en compréhension de } \\
\text { textes }\end{array}$ \\
\hline $\begin{array}{l}\text { Comprendre les questions } \\
\text { Comprendre les documents }\end{array}$ & 19 & 12 \\
Meilleure lecture & 11 & 7 \\
Meilleure compréhension & 10 & 5 \\
\hline
\end{tabular}

\section{Tableau 4}

Fréquence des liens entre les domaines de l'bistoire et du français mentionnés chez la sous-population des élèves en difficultés en rédaction de textes.

\begin{tabular}{lll}
\hline $\begin{array}{l}\text { Liens mentionnés entre les } \\
\text { domaines du français et de } \\
\text { l'histoire }\end{array}$ & $\begin{array}{l}\text { Fréquence du code chez l'ensemble } \\
\text { des répondants }\end{array}$ & $\begin{array}{l}\text { Fréquence du code chez les élèves } \\
\text { déclarant avoir des difficultés en } \\
\text { rédaction de textes }\end{array}$ \\
\hline $\begin{array}{l}\text { Structure de textes } \\
\begin{array}{l}\text { Mieux répondre à la question } \\
\text { longue }\end{array}\end{array}$ & 11 & 6 \\
\hline
\end{tabular}

\section{Les limites de la recherche}

La recherche que nous avons ici exposée comportait quelques limites : il s'agit, en effet des déclarations que les élèves ont faites dans le cadre d'un questionnaire anonyme. Il s'agissait d'un outil qui nous permettait d'esquisser les représentations des participants sur l'échec qu'ils avaient vécu. Nous avons choisi, considérant, premièrement, le sujet sensible du constat d'échec (obtenu quelques jours avant la collecte de données) et, deuxièmement, la population ciblée faisant partie d'un milieu provisoire, de nous contenter des déclarations des répondants sans leur demander de preuves de ce qu'ils avançaient (tel qu'un bulletin). Cela nous limite donc à une évaluation des compétences de l'apprenant dans le domaine du français par l'apprenant lui-même, cette évaluation étant basée, en partie, sur une appréciation personnelle des résultats obtenus durant l'année scolaire. Cela étant clarifié, nous 
considérons que les résultats obtenus vont dans le même sens que les conclusions d'autres recherches ayant étudié le lien entre lecture, écriture et histoire (Cartier et Martel, 2014, Martel, 2012, 2014; Monte-Sano, 2011; Nokes, 2013; Wineburg, 1991, 2001; Wineburg et Martin, 2004; Wineburg et al., 2011) tout en y apportant un nouvel éclairage : celui du lien entre les domaines d'histoire et du français selon les élèves ayant obtenu un constat d'échec en histoire.

\section{Discussion}

Si 62,7 \% des répondants évaluent avoir des difficultés dans la compétence de compréhension de textes dans le domaine du français (et donc possiblement dans la maîtrise du savoir-lire), il est envisageable que ces difficultés ressurgissent lorsque vient le temps de comprendre et d'analyser un document écrit, considérant qu'en histoire, l'apprenant doit, en plus, chercher à comprendre la signification et l'impact historique du document. Dans le cadre de cette recherche, nous constatons que peu d'élèves tracent des liens entre les savoir-faire développés dans le domaine du français et les savoir-faire sollicités lors de l'épreuve unique. Aucun participant n'a mentionné l'idée d'avoir des preuves pour soutenir son argumentation ou n'a tracé un pont avec le texte argumentatif dont la structure est étudiée en français au deuxième cycle du secondaire. L'analyse des réponses des participants nous permet seulement d'esquisser un lien entre les éléments pouvant compliquer et entraver la démonstration des compétences du domaine de l'histoire transitant par les savoirs-lire et écrire et leur échec en histoire. En effet, il aurait été fortement improbable qu'un élève du secondaire mentionne spontanément avoir des difficultés avec les inférences. Toutefois, l'importance relative pour les participants de bien comprendre les questions de l'épreuve unique (19 participants sur 97) pourrait être associée à des difficultés à faire des inférences ou encore avec des difficultés avec les diverses médiations en place dans l'épreuve unique. Il faudrait, pour établir avec certitude ce lien, une seconde étude axée particulièrement sur ce sujet. Dans le cadre de notre étude exploratoire du milieu des cours d'été, nous soulevons toutefois la possibilité que les participants ayant évoqué des difficultés en rédaction de textes (46,1\% des participants) puissent avoir de la difficulté avec le niveau d'abstraction requis par l'épreuve unique ainsi qu'avec les spécificités textuelles du domaine de l'histoire et que les participants ayant des difficultés en compréhension de textes risquent d'être particulièrement sensibles aux difficultés à construire les concepts en histoire, à faire des inférences et à introduire des erreurs dans les diverses médiations en place dans l'épreuve unique.

Certaines recherches ont déjà étudié les stratégies de lecture efficaces dans le domaine du français (Duffy, Roehler et Herrmann, 1988; Falardeau et Gagné, 2012). Au moment d'accomplir l'épreuve unique ministérielle en histoire, les élèves ont, pour la plupart, été familiarisés, en français (et dans d'autres domaines $^{9}$ ), avec diverses stratégies de compréhension de lecture. Dans ce contexte, il pourrait être intéressant d'amener l'apprenant à réinvestir et à adapter ces stratégies dans le cours d'histoire. Le lien que nous avons pu esquisser dans le présent article entre les difficultés en compréhension de lecture et la démonstration des compétences historiques nous prouve qu'il ne faut pas négliger l'analyse proprement textuelle des documents dans les classes d'histoire. Nokes (2013), Wineburg (Wineburg, 1991; Wineburg et Martin, 2004; Wineburg et al., 2011), Monte-Sano (2011) et Martel (Cartier et Martel, 2014; Martel, 2012) concluaient dans le même sens. 
Si nous analysons la question à réponse élaborée de l'épreuve unique, on peut s'apercevoir qu'elle est un genre littéraire en soi, à mi-chemin entre la dissertation historique et le texte argumentatif ${ }^{10}$. La préparation à cette production écrite ne devrait pas différer de celle des productions écrites du domaine du français : c'est par l'analyse, l'observation et la pratique que l'apprenant va pouvoir développer sa capacité à rédiger ce nouveau genre. L'analyse à laquelle nous référons est celle de la grille de correction de la question à réponse élaborée que le ministère de l'Éducation diffuse au grand public chaque année (pour l'examen de 2017, voir : Ministère de l'Éducation, de l'Enseignement supérieur et de la Recherche, 2016). L'étape de l'observation, mentionnée dans les recherches de Blain (1995) ou par Blaser et Chartrand (2009), réfère, quant à elle, à l'étude par les élèves de divers textes répondant aux critères de ce genre textuel et permettant de comprendre la structure attendue. On pourrait aussi réinvestir, dans le domaine de l'univers social, des exercices de coécriture ou d'échange de copie pour une première correction informelle, des stratégies étudiées par Allal (2015) et Prince (2013) qui permettent l'apprentissage et encouragent le développement de l'argumentation à l'aide d'échanges entre participants. L'échange avec les pairs peut aussi être intégré très tôt dans le processus d'écriture, permettant de discuter et de valider l'interprétation à donner aux documents historiques et à solidifier ou à modifier (si besoin est) sa compréhension tel que Leith (2011) le promeut. Tous ces exercices d'inspiration socioconstructiviste sont particulièrement intéressants pour le développement de la rigueur du raisonnement.

Bien que la formation initiale des enseignants d'histoire n'inclue habituellement pas de familiarisation avec les outils scripturaux, la présence de questions à réponse élaborée dans l'épreuve unique implique que les enseignants d'histoire ont à former leurs élèves à produire des textes de nature historique. Et même s'il peut être tentant de se décharger de cette tâche sur les épaules de l'enseignant de français, comme Hall (2005) l'a documenté, il ne faut pas oublier que la formation aux genres littéraires spécifiques à chaque domaine d'études appartient à l'enseignant spécialisé dans ce domaine (Blaser et Chartrand, 2009; Chartrand et Blaser, 2007) : les dissertations historiques pour les enseignants d'histoire, les rapports de laboratoire pour les enseignants des sciences, etc. Des partenariats avec les enseignants du domaine du français pourraient toutefois faciliter la création de liens entre ces deux disciplines (Blaser et Chartrand, 2009).

\section{Conclusion}

Les résultats de cette étude nous amènent à nous inquiéter de la situation des élèves en difficulté dans le domaine du français. En effet, à cause d'une mauvaise maîtrise des savoir-lire et savoirécrire mobilisables dans d'autres domaines d'études que le français, ils semblent être désavantagés lorsque vient le moment de démontrer leurs compétences dans le domaine de l'histoire. C'est ce que Chevallard (1985) appelait les notions protodisciplinaires : des notions dont la maîtrise implicite (dans le cas présent : un certain niveau de savoir-lire et de savoir-écrire) est essentielle pour démontrer ses compétences dans un autre domaine (ses compétences en histoire). Avec la formule actuelle de l'épreuve unique, il est très difficile de nous assurer qu'un échec en histoire ne soit pas plutôt la conséquence d'une mauvaise maîtrise des outils sémiotiques ${ }^{11}$. Serait-il possible qu'un apprenant puisse maîtriser les compétences du domaine de l'histoire sans être apte à les démontrer à cause d'une faible maîtrise des outils sémiotiques? Les difficultés dans le domaine du français semblent ressurgir dans le domaine de 
l'histoire, pouvant contribuer à développer, chez l'apprenant, un rapport négatif aux savoirs (Charlot, 1997; Chevallard, 1985; Lamine, 2000) et, peut-être même, de l'impuissance apprise (Johnson, 1981; Nougaro et Vera, 1997). Considérant que l'histoire n'est pas la seule discipline transitant par une évaluation ministérielle nécessitant un bon niveau de maîtrise du savoir-lire et du savoir-écrire, ne faudrait-il pas s'interroger sur l'impact, pour les apprenants en difficulté dans le domaine du français, de notre mode d'évaluation?

\section{Notes}

1 Une réalité qui risque de ne plus être d'actualité avec la refonte du programme d'histoire entraînant l'évaluation de toutes les compétences du programme lors de l'épreuve unique. Dans la version de 2007 du programme, nétait évaluée, pour des raisons pratiques, que la compétence « interpréter les réalités sociales à l'aide de la méthode historique », alors que les compétences « interroger les réalités sociales dans une perspective historique » et « consolider l'exercice de sa citoyenneté à l'aide de l'histoire » étaient laissées pour compte.

2 Compétence telle que formulée dans le Programme de formation de l'école québécoise : enseignement secondaire deuxième cycle de 2007 (Ministère de l'Éducation, du Loisir et du Sport, 2007). Avec la refonte du programme d'histoire (Ministère de l'Éducation et de l'Enseignement supérieur, 2016), il semblerait que les compétences du deuxième cycle du secondaire seront toutes évaluées lors de l'épreuve unique. Au moment d'écrire ces lignes, le dossier est encore sous discussion.

3 Notons que cette application artificielle de la méthode historique dans l'épreuve unique entraîne l'apprenant à répondre à une question en développant un argumentaire sans recevoir plus de points pour avoir ciblé correctement les documents les plus pertinents. Éthier, Cardin et Lefrançois l'expliquaient en ces mots : "De notre point de vue, l'épreuve unique mime l'analyse de documents, dans des conditions qui font que la "bonne" réponse ne repose pas sur une application d'une démarche historienne ou du sens critique ni même sur une compréhension réelle des concepts ou phénomènes abordés. Il s'agit plutôt, pour paraphraser Warren, de déterminer des causes et des effets ou de mettre en relation des faits à partir de documents épars.» (2014, p. 169).

4 La refonte du programme d'histoire laisse entendre qu'il y aura dorénavant deux questions à réponse élaborée reposant toutes les deux sur l'analyse de documents historiques : une évaluant toujours la rigueur du raisonnement, mais plus courte que la formule connue actuellement, et une nouvelle question évaluant la nouvelle compétence « caractériser une période de l'histoire du Québec et du Canada » (Ministère de l'Éducation et de l'Enseignement supérieur, 2016).

5 On mobilise un savoir-écrire dans l'épreuve unique de « Science et technologie et Applications technologiques et scientifiques » qui ne s'exprime pas de la même façon que celui de l'épreuve de mathématiques ou que celui de l'ÉUM.

6 Nous avons aussi documenté la prépondérance du TDAH, des troubles dys, des élèves travaillant durant l'année scolaire et nous avons documenté le rapport au(x) savoir(s) de cette population (Pageau, 2016).

7 Appellation permettant de regrouper la dyslexie, la dysorthographie, la dyscalculie, la dyspraxie, etc.

8 Nous n'avons pas demandé de preuves ou de bulletins antérieurs puisque notre objectif, dans cette recherche, était de documenter les représentations des élèves sur leurs difficultés.

9 Nous pensons, entre autres, aux mises en situation en mathématiques où l'apprenant doit aller chercher l'information pour résoudre le problème demandé ou bien, en anglais, où la deuxième compétence touche à la compréhension de textes.

10 Les prototypes d'épreuve suivant la refonte du programme d'histoire et de l'épreuve de troisième secondaire indiquent qu'il y aura dorénavant deux questions à réponse élaborée : une évaluant toujours la rigueur du raisonnement, et une nouvelle question évaluant la nouvelle compétence « caractériser une période de l'histoire du Québec et du Canada » à l'aide du critère « représentation cohérente d'une période de l'histoire du Québec et du Canada » (Ministère de l'Éducation et de l'Enseignement supérieur, 2017b). Dans ce cas, notre argumentation tient d'autant que l'enseignant d'histoire aura alors la charge de faire comprendre les deux structures requises pour ces deux types de textes. 
Les outils sémiotiques réfèrent aux outils employés pour communiquer par l'oral et l'écrit.

\section{Références}

Allal, L. (2015). Le rôle de la co-régulation dans des activités de production textuelle. Lettrure, (3), 1-14. Repéré à https://www.ablf.be/lettrure/lettrure-3/le-role-de-la-co-regulation-dans-des-activites-de-production-textuelle

Blain, R. (1995). Discours, genres, types de textes, textes...De quoi me parlez-vous? Québec français, (98), 22-25. Repéré à https://www.erudit.org/fr/revues/qf/1995-n98-qf1229585/44277ac/

Blaser, C. (2007). Fonction épistémique de l'écrit : pratiques et conceptions d'enseignants de sciences et d'histoire du secondaire (Thèse de doctorat, Université Laval, Québec). Repéré à http://hdl.handle.net/20.500.11794/19039

Blaser, C. et Chartrand, S.-G. (2009). Étayer des activités de lecture et d'écriture dans toutes les disciplines scolaires. Québec français, (154), 114-116. Repéré à https://www.erudit.org/fr/revues/qf/2009-n154-qf1095144/1831ac/

Blaser, C., Lord, F., Giraldeau, R. et Trudeau, M. P. (2014). Questions et consignes de trois manuels scolaires d'Histoire et éducation à la citoyenneté sous la loupe. Enjeux de l'univers social, 10(1), 12-15.

Cartier, S. C. et Martel, V. (2014). Apprendre par la lecture (APL) en univers social au primaire et au secondaire. Enjeux de l'univers social, 10(1), 8-11.

Charlot, B. (1997). Du rapport au savoir : éléments pour une théorie. Paris : Anthropos.

Chartrand, S.-G. (2009). Compétences à mobiliser pour la compréhension et l'interprétation de manuels d'histoire du secondaire au Québec. Revue des sciences de l'éducation, 35(2), 37-58. http://dx.doi.org/10.7202/038728ar

Chartrand, S.-G. et Blaser, C. (2007). Quelles sont les pratiques enseignantes autour de l'écriture en histoire et en sciences? Enquête dans les classes du secondaire au Québec. Formation et profession, 13(2), 33-36. Repéré à http://www.crifpe.ca/download/verify/127

Chevallard, Y. (1985). La transposition didactique : du savoir savant au savoir enseigné. Grenoble : La Pensée sauvage.

Ciardiello, A. V. (2002). Helping adolescents understand cause / effect text structure in social studies. The Social Studies, 93(1), 31-36. http://dx.doi.org/10.1080/00377990209599877

Demers, S., Lefrançois, D. et Éthier, M.-A. (2014). Lire l'histoire locale : du patrimoine bâti aux sources écrites. Enjeux de l'univers social, 10(1), 24-29.

Déry, C. (2016). Description et analyse des postures épistémologiques sous-tendues par l'épreuve unique ministérielle en Histoire et éducation à la citoyenneté (Mémoire de maîtrise, Université de Montréal). Repéré à https://papyrus.bib.umontreal.ca/xmlui/handle/1866/18600

Déry, C. (2017). Description et analyse des postures épistémologiques sous-tendues par l'épreuve unique ministérielle de quatrième secondaire en Histoire et éducation à la citoyenneté. McGill Journal of Education, 52(1), 149-172. http://dx.doi.org/10.7202/1040809ar

Duffy, G. G., Roehler, L. R. et Herrmann, B. A. (1988). Modeling mental processes helps poor readers become strategic readers. The Reading Teacher, 41(8), 762-767.

Éthier, M.-A., Cardin, J.-F. et Lefrançois, D. (2014). Leur programme et le nôtre. Bulletin d'histoire politique, 22(3), 165178. http://dx.doi.org/10.7202/1024154ar

Falardeau, É. et Gagné,J.-C. (2012). L’enseignement explicite des stratégies de lecture : des pratiques fondées par la recherche. Enjeux, (83), 91-120.

Fortin, F., Côté, J. et Filion, F. (2006). Fondements et étapes du processus de recherche. Montréal, QC : Chenelière Éducation.

Grenier, S., Jones, S., Strucker, J., Murray, T. S., Gervais, G. et Brink, S. (2003). Enquête internationale sur l'alphabétisation des adultes. L'apprentissage de la littératie au Canada: Constatations tirées de l'Enquête internationale sur les compétences en lecture. Ottawa, ON : Statistique Canada.

Hall, L. A. (2005). Teachers and content area reading: Attitudes, beliefs and change. Teaching and Teacher Education, 21(4), 403-414. http://dx.doi.org/10.1016/j.tate.2005.01.009

Johnson, D. S. (1981). Naturally acquired learned helplessness: the relationship of school failure to achievement behavior, 
attributions, and self-concept. Journal of Education E Psychology, 73(2), 174-180.

http://dx.doi.org/10.1037/0022-0663.73.2.174

Kendeou, P., van den Broek, P., Helder, A. et Karlsson, J. (2014). A cognitive view of reading comprehension: Implications for reading difficulties. Learning Disabilities Research and Practice, 29(1), 10-16. http://dx.doi.org/10.1111/1drp.12025

Lamine, B. A. M. (2000). Pertinence et limites de la notion de « rapport au savoir » en didactique des sciences. Dans A. Chabchoub (dir.), Rapports aux savoirs et apprentissage des sciences (p. 187-194). Tunis: ATRD.

Laparra, M. (1991). Problèmes de lecture posés par l'écriture des textes historiques à visée didactique. Pratiques, (69), 97-124.

Leith, É. (2011). Le journal dialogué et le cercle de lecture : des outils au service d'une lecture interactive. Québec français, (160), 50-51. Repéré à http://id.erudit.org/iderudit/61623ac

Makdissi, H. et Boisclair, A. (2008). L'organisation discursive du rappel de récit chez l'enfant d'âge préscolaire. Archives de psychologie, 73(284-285), 51-79.

Martel, V. (2012). Lire en histoire : Mieux lire les textes informatifs et s'ouvrir aux œuvres littéraires. Traces, 51(1), 18-24.

Martel, V. (2014). Lire en univers social : tour d'horizon et introduction de ce dossier spécial. Enjeux de l'univers social, 10(1), 4-19.

Ministère de l'Éducation et de l'Enseignement supérieur. (2013). Examens et épreuves : Tableau 4 - Résultats par matière, selon certaines variables, pour la session de juin 2012. Québec, QC : Ministère de l'Éducation et de l'Enseignement supérieur.

Ministère de l'Éducation et de l'Enseignement supérieur. (2014). Examens et épreuves : Tableau 4 - Résultats par matière, selon certaines variables, pour la session de juin 2013. Québec, QC : Ministère de l'Éducation et de l'Enseignement supérieur.

Ministère de l'Éducation et de l'Enseignement supérieur. (2016). Programme de formation de l'école québécoise, Histoire du Québec et du Canada, Enseignement secondaire $2^{e}$ cycle, $3^{e}$ et $4^{e}$ secondaire [Diffusion restreinte, version du 6 octobre 2016]. Québec, QC : Ministère de l'Éducation et de l'Enseignement supérieur.

Ministère de l'Éducation et de l'Enseignement supérieur. (2017a). Examens et épreuves. Québec, QC : Ministère de l'Éducation et de l'Enseignement supérieur.

Ministère de l'Éducation et de l'Enseignement supérieur. (2017b). Document d'information pour le prototype d'épreuve de juin 2017 (3e année du secondaire). Québec, QC : Ministère de l'Éducation et de l'Enseignement supérieur.

Ministère de l'Éducation, de l'Enseignement supérieur et de la Recherche. (2015a). L'apprentissage de l'orthographe au primaire et au secondaire. Bulletin Objectif Persérérance et Réussite, (13), 1-13. Repéré à http://www.education.gouv.qc.ca/fileadmin/site web/documents/PSG/recherche evaluation/BulletinObjectif 13.pdf

Ministère de l'Éducation, de l'Enseignement supérieur et de la Recherche. (2015b). Examens et épreuves : Tableau 4 - Résultats par matière, selon certaines variables, pour la session de juin 2014. Québec, QC : Ministère de l'Éducation, de l'Enseignement supérieur et de la Recherche.

Ministère de l'Éducation, de l'Enseignement supérieur et de la Recherche. (2015c). Prévisions de l'effectifétudiant au préscolaire, au primaire et au secondaire - Prévisions pour l'ensemble du Québec. Repéré à http://www.education.gouv. qc.ca/fileadmin/site web/documents/PSG/statistiques info decisionnelle/previsions provinciales.pdf

Ministère de l'Éducation, de l'Enseignement supérieur et de la Recherche. (2016). Épreuve unique : Document d'information - Juin 2017 - Juillet 2017 - Janvier 2018. Québec, QC : Ministère de l'Éducation, de l'Enseignement supérieur et de la Recherche.

Ministère de l'Éducation, du Loisir et du Sport. (2007). Histoire et éducation à la citoyenneté. Dans Programme de formation de l'école québécoise : enseignement secondaire deuxième cycle (p. 109). Québec, QC : Ministère de l'Éducation, du Loisir et du Sport.

Mialaret, G. (2004). Méthodes de recherche en sciences de l'éducation. Paris : Presses universitaires de France.

Moniot, H. (1993). Didactique de l'histoire. Paris : Nathan.

Monte-Sano, C. (2011). Beyond reading comprehension and summary: Learning to read and write in history by focusing 
on evidence, perspective, and interpretation. Curriculum Inquiry, 41(2), 212-249. http://dx.doi.org/10.1111/j.1467-873X.2011.00547.x

Nokes, J. D. (2013). Building students' historical literacies: Learning to read and reason with historical texts and evidence. New York, NY : Routledge.

Nougaro, F. et Vera, L. (1997). Impuissance apprise et dyslexie. Rééducation orthophonique, 35(192), 95-103.

Pageau, L. (2016). À quelles causes les élèves inscrits au cours d'été en bistoire et éducation à la citoyenneté de quatrième secondaire attribuent-ils leur échec à l'épreuve unique ministérielle? (Mémoire de maîtrise, Université Laval, Québec). Repéré à http://hdl.handle.net/20.500.11794/27286

Poisson, Y. (1992). Familles de recherche qualitative. Dans La recherche qualitative en éducation (p. 21-32). Québec, QC : Presses de l'Université du Québec.

Prince, M. (2013). La réécriture accompagnée. Les cabiers de L'AQPF, 4(1), 20-24.

Savoie-Zajc, L. (2007). Comment peut-on construire un échantillonnage scientifiquement valide? Recherches qualitatives, hors série(5), 99-111. Repéré à http://www.recherche-qualitative.qc.ca/documents/files/revue/hors serie/hors serie v5/savoie zajc.pdf

Schneuwly, B. (2008). Vygotski, l'école et l'écriture. Repéré à https://archive-ouverte.unige.ch/unige:18580/ATTACHMENT01

Smidt, S. (2009). Introducing Vygotsky: A guide for practitioners and students in early years education. Londres : Routledge.

Stan, C. A. (2017). Penser le monde : l'apport de l'histoire à la compréhension des phénomènes et des enjeux sociaux du XXI ${ }^{\mathrm{e}}$ siècle. Traces, 55(3), 32-36.

Warren, J.-P. (2013). Enseignement, histoire, mémoire. Les examens d'histoire de 4 e secondaire du secteur de la formation générale au Québec (1970-2012). Revue d’histoire de l'éducation, 25(1), 31-53. Repéré à http://historicalstudiesineducation.ca/index.php/edu hse-rhe/article/view/4308

Wineburg, S. S. (1991). On the reading of historical texts: Notes on the breach between school and academy. American Educational Research Journal, 28(3), 495-519. http://dx.doi.org/10.3102/00028312028003495

Wineburg, S. (2001). Historical thinking and other unnatural acts: charting the future of teaching the past. Philadelphie, PA : Temple University Press.

Wineburg, S. et Martin, D. (2004). Reading \& rewriting history. Educational Leadership, 62(1), 42-45. Repéré à http://www.ascd.org/ASCD/pdf/journals/ed lead/el200409 wineburg.pdf

Wineburg, S., Martin, D. et Monte-Sano, C. (2011). Reading like a historian: Teaching literacy in middle and high school bistory classrooms. New York, NY : Teachers College Press.

\section{Pour citer cet article}

Pageau, L. (2018). Étude du lien entre l'échec à l'épreuve unique ministérielle en histoire et les difficultés dans le domaine du français. Formation et profession, 26(2), 35-53. http://dx.doi.org/10.18162/fp.2018.465 